\title{
Impact environnemental et évaluation de la qualité des eaux par des méthodes chimiques et biologiques « Diatomées »
}

\author{
Nouzha CHAHBOUNE ${ }^{1 *}$, Mohamed MEHDI ${ }^{1}$, Mohamed ABIDI ${ }^{2}$ et Allal DOUIRA ${ }^{1}$ \\ ${ }^{1}$ Université Ibn Tofail, Faculté des Sciences, Laboratoire de Botanique et de Protection des Plantes, \\ B. P. 133, Kénitra, Maroc. \\ ${ }^{2}$ Institut d'Hygiène, Rabat, Maroc. \\ *Auteur correspondant; E-mai: chahbounenouzha@yahoo.fr; labobotanique@gmail.com
}

\section{RESUME}

Le but de cette étude est de surveiller la qualité des eaux de la Merja Fouarat par l'utilisation des diatomées benthiques comme bio-indicateurs et des variables chimiques telles que DBO5, ammoniums, nitrites et phosphates. L'évolution spatio-temporelle des valeurs de l'Indice de pollution organique (IPO) et de l'indice diatomique Leclercq (IDL), montrent que l'eau de la Merja Fouarat est fortement polluée à cause des rejets non traités des eaux usées domestiques et industrielles. L'IDL et L'IPO se classent dans le même niveau de pollution avec un écart faible (0,27-0,3-0,5-0,2-0,3), témoin que les diatomées intègrent les variations des facteurs polluants. Par ailleurs, les espèces rencontrées possèdent un caractère alcaliphyle prononcé avec une abondance des espèces résistantes à la pollution organique.

(C) 2012 International Formulae Group. All rights reserved.

Mots clés : Diatomées, qualité de l'eau, bio-indicateur, IDL, IPO, Merja Fouarat.

\section{INTRODUCTION}

La région du Gharb au Maroc est très riche en zones humides, en particulier les lacs et les Merja. Ces écosystèmes fragiles subissent une intense pression anthropique due aux multiples rejets des eaux usées domestiques et industrielles non traitées (Benbouih et al., 2005). La Merja Fouarat (Kénitra) constitue un exemple concret de cette problématique. Elle est entourée d'agglomérations et d'usines, qui lui servent de déversoir. Les eaux de la Merja se déversent dans l'Oued Sebou qui à son tour se déverse dans l'océan Atlantique (Figure 1).

L'évaluation de degré de pollution par des méthodes chimiques laisse beaucoup à désirer, notamment face à la fluctuation des paramètres de pollution. Pour remédier à ce problème, il est nécessaire d'étudier la flore des diatomées, via l'utilisation de l'indice diatomique, car elle intègre les variations de la physico-chimie de l'eau, (Prygiel et al., 2002; Lavoie et al., 2008). Les diatomées sont d'excellents bio-indicateurs de la qualité des eaux (Winter et Duthie, 2000; Wunsam et al., 2002; Rimet et al., 2006; Lavoie et al., 2008).

Leur inventaire permet d'apprécier les niveaux de pollution organique et d'eutrophisation, (Morin et Coste, 2006). Partant de ce principe, nous proposons dans ce travail, de les utiliser comme bio-indicateurs, 
en vue d'évaluer la qualité des eaux de la Merja Fouarate. Des analyses comparatives physico-chimiques et diatomiques ont été réalisées au niveau de cinq stations, pendant trois saisons successives (été, hiver et printemps).

\section{MATERIEL ET METHODES}

Caractéristiques des sites étudiés

Située dans le plateau de la Mamora, entre la plaine argileuse plio-quaternaire du Rharb et la Meseta occidentale schistogréseuse granitique et Paléozoique, la Merja Fouarat est le siège d'une nappe phréatique située dans les sables et grés-calcaires du PlioVillafranchien à climat méditerranéen et sous influence océanique prononcée

(Subhumide en étiage, tempérée en hiver).

La Merja Fouarat est alimenté par :

- Les apports de l'Oued Fouarat qui forme la première vallée importante du plateau de la Mamora avec une source localisée à Ras-El-Aîn, à $10 \mathrm{Km}$ environ au sud de la Merja;

Les pluies abondantes et rapprochées provoquent l'exfiltration des eaux naturelles de la nappe phréatique, (Nassali et al., 2002), accompagnées des inondations de courte durée;

- Les eaux résiduaires des quartiers périphériques.

\section{Echantillonnage}

$\mathrm{Au}$ niveau des cinq stations, trois campagnes de prélèvement d'eau et de diatomées sont effectuées. Les diatomées sont prélevées par grattage de substrats naturels à l'aide d'une brosse à gros poils de nylon. Les échantillons ainsi prélevés seront fixés immédiatement au formol (10\%). Après quelques jours de décantation, les diatomées sont traitées avec l'acide nitrique sur plaque chauffante, puis montées avec du naphrax entre lame et lamelle. L'identification des diatomées selon (Krammer et Langbertalot, 1986; 1988; 1991a et b; 2000) et le comptage sous microscope optique de 450 frustules au minimum est effectué sur des préparations permanentes. Les effectifs de chaque taxon sont transformés en abondance relative. Les analyses physico-chimiques sont effectuées selon les méthodes de Rodier, 1996.

\section{Méthodes d'analyses \\ Indice de pollution organique}

L'indice de pollution organique (Leclercq, 2001) est basé sur les mesures de DBO5, ammoniums, nitrites et phosphates, réparties en cinq classes qui ont une signification biologique correspondant à des modifications typiques des peuplements de diatomées.

\begin{tabular}{llr}
\multicolumn{3}{c}{ Analyse et calcul de l'indice } \\
DBO5 & 3,7 & 4 \\
Ammonium & 1,2 & 3 \\
Nitrites & 35 & 3 \\
Phosphates & 12 & 5 \\
IPO & $4+3+3+5 / 4=3,75$
\end{tabular}

\section{Interprétation de l'indice}

5,0 - 4,6 pollution nulle

4,5 - 4,0 pollution faible

3,9 - 3,0 pollution modérée

$2,9-2,0$ pollution forte

$1,9-1,0$ pollution très forte

\section{Calcul de l'indice diatomique Leclercq}

Pour l'évaluation biologique de la qualité des eaux de la Merja, on a utilisé l'indice diatomique Leclercq (IDL). Les valeurs indicielles établies par, sont généralement réparties en cinq classes correspondant chacune à un niveau de pollution. Cette méthode se fonde sur la formule suivante :

Indexsaprobique $=\frac{\sum_{x=1}^{n} A x \cdot V x \cdot I x}{\sum_{n=1}^{n} A x \cdot I x}$

$\mathrm{n}=$ nombre de taxons du relevé,

$\mathrm{A}=$ abondance relative (en \%) de chaque taxon,

$\mathrm{V}=$ valence saprobique de chaque taxon,

$\mathrm{I}=$ valeur indicatrice de chaque taxon. 


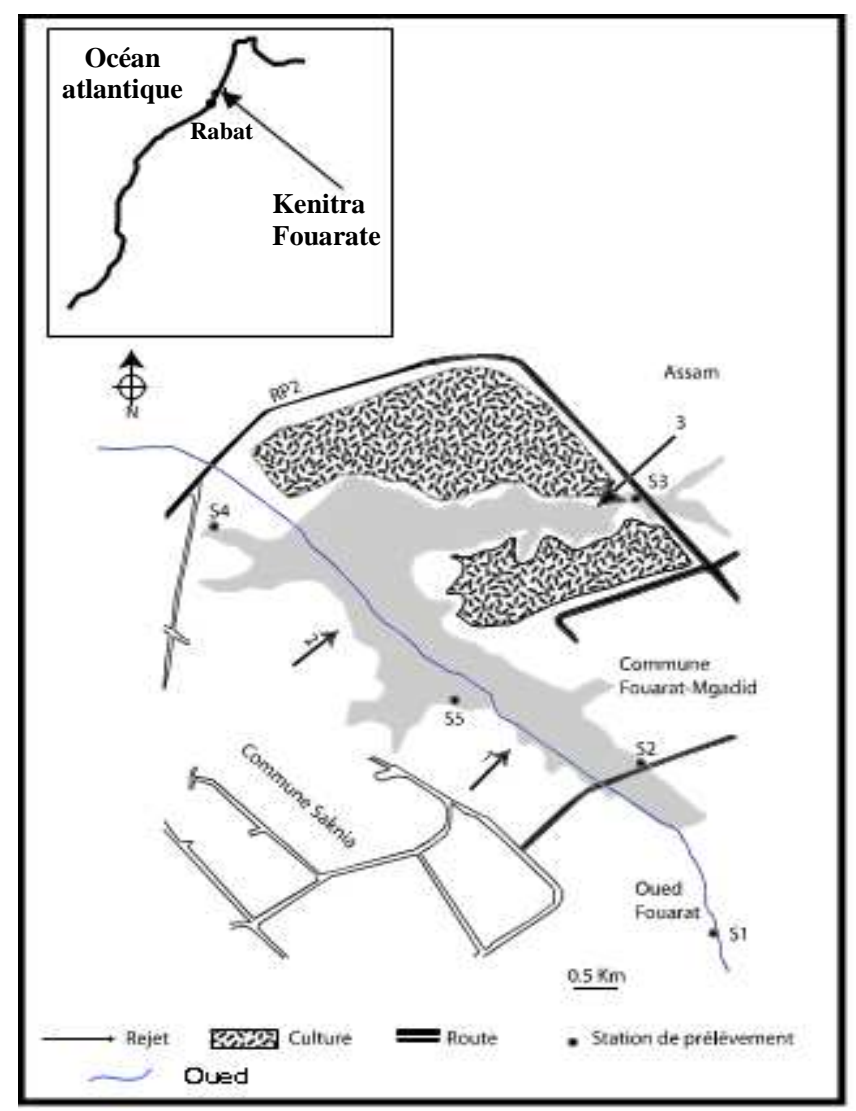

Figure 1: Situation géographique des stations de prélèvements. S1 : Station de référence (Oued Fouarat). S2, S3, S4 et S5 : Stations le long de la Merja Fouarat.

\section{RESULTATS ET DISCUSSION Indice de pollution organique (IPO)}

L'IPO indique un état de pollution modérée au niveau des stations $\mathrm{S} 1$ et $\mathrm{S} 2$. (Tableaux 1 et 2) et passe d'un état de pollution forte (station 3 et 4 ) à très forte (station 5). Les valeurs de cet indice s'expliquent par les teneurs très élevées en ammonium et en phosphate (Benzizoune et al., 2004; Benbouih et al., 2005).

L'évolution des valeurs de l'IPO le long des stations (Figure 2) montre que cet état dégradé de la qualité des eaux de la Merja est dû aux rejets des eaux usées domestiques et industrielles provenant des agglomérations de Saknia, Fouarat, Mgadid et Assam (Benbouih et al., 2005).

La campagne d'hiver présente un état de pollution relativement plus important par rapport à celle du printemps. Cela s'explique par le lessivage des terrains agricoles en hiver (Derradji et al., 2007) d'une part et l'assimilation des nutriments par les végétaux en pleine période de végétation (le printemps) d'autre part.

\section{Analyse diatomique}

Au niveau de la Merja Fouarat et au cours des 3 saisons (hiver, printemps et été), les résultats de nos comptages montrent l'abondance d'une population diatomique (Figure 3) caractéristique de milieux fortement pollués et très minéralisés (Chahboune et al., 2011). En effet, la présence de Nitzschia palea (Inthasotti, 2011), Gomphonema parvulum (Thi Thuy et al., 2007), Cyclotella meneghiniana (Straub et al., 2003), Amphora veneta Kutzing, Navicula 
subminuscula, Navicula veneta (Fawzi et al., 2001) souligne l'existence d'une pollution organique.

La flore diatomique est particulièrement pauvre en Pinnularia et d'autres genres acidophiles qui ne sont représentés que par les espèces les plus tolérantes et indifférentes au $\mathrm{pH}$, cas de Pinnularia gibba Ehrenberg (Fawzi et al., 2001). Par contre, toutes les espèces dominantes possèdent un caractère alcaliphile prononcé.

La concentration des chlorures est généralement inférieure à $500 \quad \mathrm{mg} / \mathrm{l}$ (Benzizoune et al., 2004; Chahboune et al., 2011) ce qui favorise l'apparition d'une population d'eau douce accompagnée de certaines espèces euryalines.

\section{Indice diatomique Leclercq}

Niveau typologique

Pour déterminer le niveau typologique des eaux de la Merja, on se réfère d'abord aux espèces sensibles à la pollution sinon à d'autres en cas où celles-ci disparaissent par action anthropique.

Le type d'eau potentielle au niveau de la Merja et Oued Fouarat est eutrophe (Chahboune et al., 2011). Cette situation est confirmée par la présence d'un assemblage des espèces sensibles et très sensibles telle que Cymbella sinuata Gregory (Pierre 1994), Navicula cryptotenella Lange-Bertalot (Rimet et al., 2006), Amphora pediculus (Kutzing) Grunow (Lavoie et al., 2008) et Cocconeis placentula (Ehenberg) Grunow var. euglypta (Montesanto et al., 1999; Stancheva et al., 2007).

Dans les stations polluées (3, 4 et 5 ), les espèces sensibles à la pollution organique disparaissent au profit des espèces résistantes, tout en gardant le caractère eutrophe: Navicula veneta Kutzing, Navicula subminiscula Manguin, Cyclotella meneghiniana Kutzing (Rimet et al., 2006) et Amphora veneta Kutzing (Marosi et al., 2006).

Pendant la saison du printemps et au niveau de la station 2, l'abondance de l'espèce
Achnanthes minutissima Kutzing var. saprophila Kobayasi et Mayama n'est pas significative quant à l'indication typologique (Momeu et al., 2005) puisque celle-ci se rencontre aussi bien dans les eaux oligo-méso et eutrophes.

\section{Niveau de pollution organique au niveau de la Merja Fouarat}

L'IDL moyen indique une dégradation importante de la qualité des eaux au niveau des stations 2, 3, 4 et 5 (Tableau 3) durant la saison d'hiver, printemps et été avec un écarttype très faible, ce qui montre que les diatomées et les indices diatomiques intègrent la qualité des eaux sur des périodes relativement longues (Fawzi et al., 2001), chose confirmée par l'abondance des espèces résistantes et très résistantes à la pollution organique.

En effet, on note une abondance relativement élevée des espèces résistantes à la pollution organique de caractère eutrophe au niveau de:

- La station 2, $22.3 \%$ de Navicula goeppertiana, $5.3 \%$ de Gomphonema parvulum, $2.6 \%$ de Navicula cryptocephala (Rimet et al., 2006), 6.1\% de Cyclotella meneghiniana (Straub et al., 2003) et $3.5 \%$ de Navicula subminuscula (Rimet et al., 2006).

- La station 3, correspond aussi a une mosaïque d'espèces diatomiques avec une abondance de $24.9 \%$ de Nitzschia palea (Inthasotti, 2011), 6.9\% de Gomphonema parvulum (Thi Thuy et al., 2007), 19.1\% d'Amphora veneta (Marosi et al., 2006), 11\% de Nitzschia perminuta, 4.5 de Navicula cryptocephala, $5.3 \%$ de Cyclotella meneghiniana (Straub et al., 2003), 5.3\% de Navicula veneta, et $6.9 \%$ de Navicula confervacea.

- La station 4, marque une abondance très élevée de Navicula cryptocephala (45.9\%), 5.1\% de Cyclotella meneghiniana (Straub et al., 2003), 6.6\% de Navicula subminuscula, $13.4 \%$ de Navicula veneta (Fawzi et al., 2001), 5\% de Nitzschia perminuta et $14.7 \%$ de Navicula confervacea. 
- $\quad$ La station 5 est caractérisée par un assemblage de $52.1 \%$ de Navicula Subminuscula, $11.9 \%$ de Nizschia capitellata, $15.3 \%$ de Nitzschia palea (Inthasotti, 2011), $37.8 \%$ de Gomphonema parvulum (Thi Thuy et al., 2007) et (Rimet et al., 2006) et $16.3 \%$ de Navicula accomoda.

Pour l'Oued Fouarat (Station 1), la faible valeur de l'IDL peut s'expliquer par le lessivage des terrains agricoles en hiver et par l'évaporation des eaux en été, ce qui augmente la concentration de l'eau en éléments nutritifs et par conséquent augmente la prolifération des espèces résistantes à la pollution organique. $\mathrm{Au}$ printemps, l'assimilation des nutriments (nitrates et phosphates) favorise la clarté et la bonne qualité des eaux, ce qui permet le développement des espèces sensibles à la pollution organique et de ce fait, la valeur de l'indice diatomique augmente. Le caractère topographique de terrain (pente) améliore la qualité des eaux, comme c'est le cas de la Station 2 où les eaux courantes et oxygénées favorisent la dégradation de la matière organique par les bactéries et permettent la prolifération des espèces hypertrophes comme Amphora pediculus (12.7\%) (Lavoie et al., 2008 ) et Gomphonema truncatum (1.6\%) sensibles à la pollution organique et responsables de l'augmentation de l'IDL (Inthasotti, 2011).

La méthode d'échantillonnage peut fausser les résultats de l'analyse biologique. En effet, l'échantillonnage des diatomées sur des algues filamenteuses peut ne pas être significatif vis-à-vis de l'IDL. La Station 3, par exemple présente une forte pollution au printemps et une très forte pollution en hiver alors qu'en été, son IDL est surestimé du fait que nous avons effectué des prélèvements sur des algues filamenteuses. $\mathrm{Si}$ on retire la Cocconeis placentula et si on recalcule les pourcentages, les espèces résistantes seront dominantes, avec un niveau de pollution fort à très fort et un IDL très bas.

\section{Niveau d'hypertrophisation}

En hiver et printemps, les stations 1 et
2 marquent la présence d'espèces Achnanthes lanceolata (Breb.), Fragilaria ulna Lange Bertalot (Pierre, 1994), Navicula viridula (Kutzing) Ehrenberg significatives d'une eutrophisation (Hamaidi et al., 2009), chose inverse au niveau des autres stations, malgré la haute teneur en phosphate et en nitrate. Ici la surcharge en matière organique élimine la prolifération des diatomées indicatrices d'eutrophisation.

\section{Evolution spatio-temporelle de L'Indice Diatomique Leclercq (IDL)}

L'analyse des valeurs de l'IDL montre des fluctuations spatio-temporelles de la qualité des eaux au niveau de la station 1 et 2 . En effet, la Station 1 présente une pollution organique faible au printemps, modérée en hiver à forte en été, ce qui montre que l'augmentation du débit de l'eau en hiver et de la photosynthèse au printemps améliore la qualité de l'eau.

La Station 2 est l'une des stations les plus polluées de la Merja, l'IDL est de l'ordre de 2,7 en hiver à cause des eaux usées non traitées du quartier Fouarat Mgadid. Mais la présence d'une pente et l'augmentation du débit de l'eau au printemps augmentent l'oxygénation de l'eau et favorisent la prolifération de Achnanthes minutissima, espèce sensible à la pollution organique, d'où une augmentation de l'IDL (3.2).

Au niveau des Stations 3,4 et 5, l'IDL indique le même niveau de pollution (pollution forte) pour les trois saisons. L'IDL évolue d'une manière descendante en passant d'une pollution modérée au niveau de l'Oued Fouarat à une pollution très forte au niveau de la commune Saknia. Cette dégradation progressive de la qualité de l'eau, liée principalement aux rejets des eaux usées domestiques et industrielles non traitées (Benbouih, 2005; Chahboune et al., 2011) entraine une diminution des capacités d'autoépuration de la Merja (Benzizoune, 2004).

\section{Comparaison entre l'IDLm et l'IPOm}

Pour les cinq stations, l'IPOm se classe 
dans les mêmes niveaux de pollution que l'IDLm (Figure 4) avec un écart respectif de $0,27,0,3,0,5,0,2,0,38$. Les deux indices évoluent d'une façon régressive et montrent une dégradation progressive de la qualité de l'eau.

Dans toutes les stations, et pendant les trois saisons, les indices sont en transition entre altération faible à l'Oued Fouarat (Station 1) en amont de la Merja et forte autour de cette dernière $\left(\begin{array}{lll}2 & \text { à } & 5\end{array}\right)$. L'autoépuration ne se marque pas à l'exception de la Station 1 qui présente une bonne qualité d'eau en pleine période de végétation, cette situation est expliquée par l'assimilation des nutriments et par la saturation des eaux en oxygène grâce à la photosynthèse algale, d'où une autoépuration meilleure et un retour aux conditions écologiques et aux peuplements originels (Benzizoune, 2004).

L'IDL et l'IPO montrent que l'eau de la Merja est de mauvaise qualité. L'évolution similaire de ces deux indices explique que les indices diatomiques européenne traduisent de façon satisfaisante la pollution organique (Coste et al., 2008) et que ces indices réagissent bien dans les régions du Maroc (Fawzi et al., 2001).

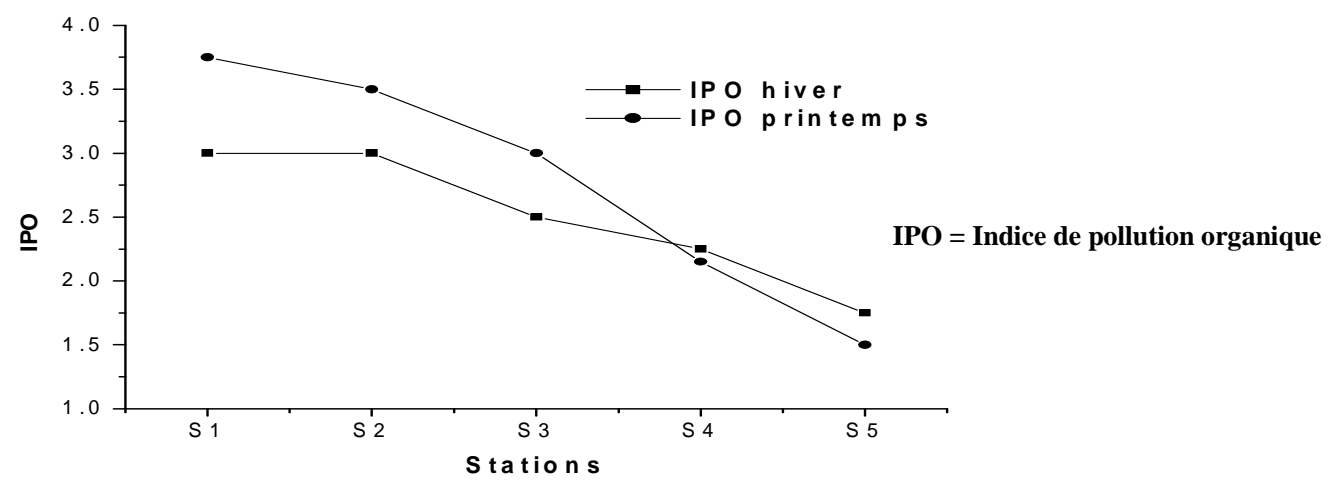

Figure 2: Evolution spatio-temporelle de l'indice de pollution organique.

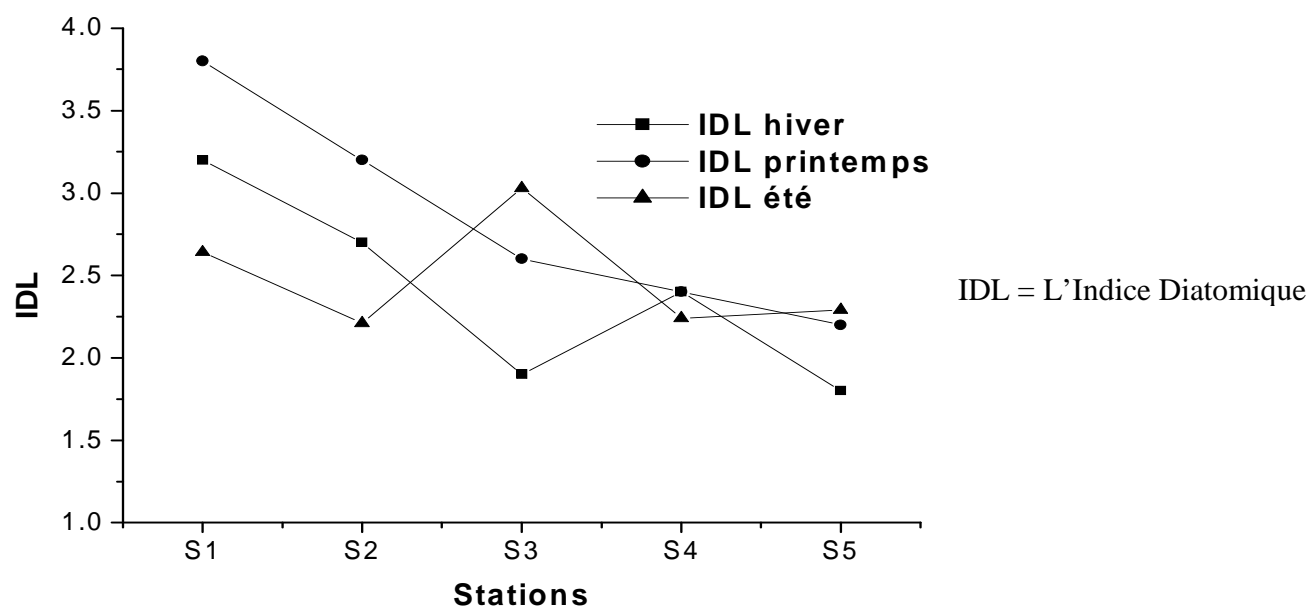

Figure 3 : Evolution spatio-temporelle de l'indice diatomique Leclercq (IDL). 


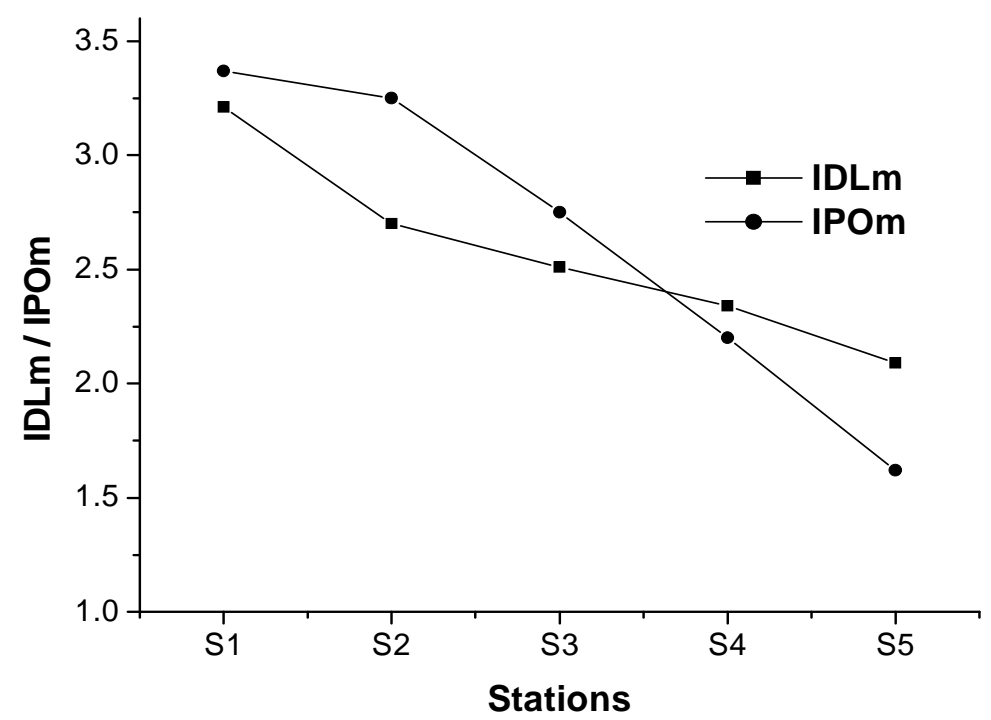

Figure 4: Comparaison de l'indice diatomique Leclercq moyen et l'indice de pollution organique moyen.

Tableau 1: Indice de Pollution Organique (IPO).

\begin{tabular}{ccccc}
\hline DBO5 & $\begin{array}{c}\text { Ammonium } \\
(\mathbf{m g}-\mathbf{N} / \mathbf{I})\end{array}$ & $\begin{array}{c}\text { Nitrites } \\
(\boldsymbol{\mu} \mathrm{g}-\mathbf{N} / \mathbf{I})\end{array}$ & $\begin{array}{c}\text { Phosphates } \\
(\boldsymbol{\mu} \mathrm{g}-\mathrm{P} / \mathbf{I})\end{array}$ \\
\hline 5 & $<2$ & $<0,1$ & $\leq 5$ & $\leq 5$ \\
4 & $2-5$ & $0,1-0,9$ & $6-10$ & $16-75$ \\
3 & $5,1-10$ & $1,0-2,4$ & $11-50$ & $75-250$ \\
2 & $10,1-15$ & $2,5-6,0$ & $51-150$ & $251-900$ \\
1 & $>15$ & $>6,0$ & $>150$ & $>900$ \\
\hline
\end{tabular}

Tableau 2: Résultats de l'indice de pollution organique.

\begin{tabular}{lccccc}
\hline Indices & Station 1 & Station 2 & Station 3 & Station 4 & Station 5 \\
\hline IPO hiver & 3 & 3 & 2,5 & 2,25 & 1,75 \\
Altération & modérée & modérée & forte & forte & Très forte \\
IPO printemps & 3,75 & 3,5 & 3 & 2,15 & 1,5 \\
Altération & modérée & modérée & modérée & forte & Très forte \\
\hline
\end{tabular}


Tableau 3: Indice diatomique Leclercq et niveau d'altération.

\begin{tabular}{lccccc}
\hline Station IDL & Station $\mathbf{1}$ & Station $\mathbf{2}$ & Station 3 & Station 4 & Station 5 \\
\hline IDL hiver & 3,2 & 2,7 & 1,9 & 2,4 & 1,8 \\
IDL printemps & 3,8 & 3,2 & 2,6 & 2,4 & 2,2 \\
IDL été & 2,64 & 2,21 & 3,03 & 2,24 & 2,29 \\
IDL moyen & 3,21 & 2,7 & 2,51 & 2,34 & 2,09 \\
Ecart-Type & 0,58 & 0,49 & 0,57 & 0,09 & 0,26 \\
Altération & modérée & forte & forte & forte & Très forte \\
\hline
\end{tabular}

\section{Conclusion}

Les résultats obtenus dans le cadre de cette étude ont montré une baisse assez généralisé de la qualité des eaux des 5 stations. L'étude des diatomées et l'application de l'indice diatomique Leclercq ont permis d'appréhender la qualité des eaux des cinq stations, le classement des eaux en pollution forte par l'IDL est essentiellement dû à la présence des espèces résistantes à la pollution organique. L'IPO se classe dans les mêmes niveaux de pollution que l'IDL en suivant plus ou moins son évolution avec un écart respectif de $0,27,0,3,0,5,0,2,0,38$ permettant de conclure que les diatomées intègrent les variations spatio-temporelles des facteurs polluants.

Face à cette situation écologique dégradante, la Merja Fouarat doit disposer des moyens de protection et de traitement des rejets par la mise en œuvre d un programme d'égouttage et d'installation des stations d'épurations.

\section{REFERENCES}

Benbouih H, Nassali H, Leblans M, Srhiri A. 2005. Contamination en métaux traces des sédiments du lac Fouarat (Maroc). Afrique Sciences, 1(1): 109-125.

Benzizoune S, Nassali H, Srhiri A. 2004. Etude de la cinétique d'adsorption du Phosphore en solution sur les sediments du lac Fouarat au Maroc. Larhyss Journal, 3: 171-184.
Chahboune N, Mehdi M, Douira A. 2011. Détérioration des écosystèmes aquatiques dans la Région du Gharb (Maroc): Analyse diatomique. Science Lib., 3: $\mathrm{N}^{\circ} 110808$, ISSN2111-4706

Coste M, Peres F, Le cohu R, Sellier E, , Morin S. 2008. Biodiversité des diatomées benthiques des rivières de la Réunion: floristique et biogéographie. 216p.

Derradji F, Bousnoubra H, Kherici N, Romeo M, Caruba R. 2007. Impact de la pollution organique sur la qualité des eaux superficielles dans le Nord-Est algerien. Secheresse, 18: 23-27.

Fawzi B, Chlaida M, Oubrahim S, Loudiki M, Sabour B, Bouzidi A. 2001. Application de certains indices diatomiques à un cours d'eau marocain: Oued Hassar. Science de l'Eau, 14(1): 73-89.

Hamaidi MS, Hamaidi F, Zoubiri A, Benouaklil F, Dhan Y. 2009. Etude de la Dynamique des Populations Phytoplanctoniques et Résultats Préliminaires sur les Blooms Toxiques à Cyanobactéries dans le Barrage de Ghrib (Ain Defla-Algérie). European Journal of Scientific Research, 32(3): 369-380.

Inthasotti T, Peerapornpisal Y. 2011. Investigation of Benthic Diatoms in the Area of Kham Watershed,Chiang Rai Province. Journal of the Microscopy Society of Thailand, 4(2): 98-102.

Krammer K, Langebertalot H. 1986. 
Bacillariophyceae. Tome 1: Naviculaceae. Semper Bonis Artibus; 876 p.

Krammer K, Langebertalot H. 1988. Bacillariophyceae. Tome 2: Bacillariaceae, Epithemiaceae et Surirellaceae. Semper Bonis Artibus; 596 p.

Krammer K, Langebertalot H. 1991a. Bacillariophyceae. Tome 3: Centrales, Fragilariaceae, Eunotiaceae. Semper Bonis Artibus; 576 p.

Krammer K, Langebertalot H. 1991b. Bacillariophyceae. Tome 4: Achnanthaceae. Semper Bonis Artibus; $437 \mathrm{p}$.

Krammer K, Langebertalot H. 2000. Bacillariophyceae (Tome 5). Semper Bonis Artibus. Englishand French translation of the Keys; $311 \mathrm{p}$.

Lavoie I, Hamilton P, Campeau S. 2008. Guide d'Identification des Diatomées des Rivières de l'Est du Canada. Presses de l'Université du Québec: Québec; 241 p. et 68 planches taxonomiques.

Leclercq L. 2001. Intérêt et Limites des Méthodes d'Estimation de la Qualité de l'Eau. Station Scientifique des HautesFagnes: Belgique.

Marosi BA, Kiss A, Péterfi LS. 2006. Preliminary studies on the structure of diatom communities in the ponds of the nature reserve Fânatetes Clujului (Transylvanita, Romania). Contribuţii Botanice, 41(1): 55-61

Momeu L, Budurlean M, Cristea V. 2005. Algal flora of the « valea Morii » nature reserve and surrounding area (Cluj County,Romania). Contribuţii Botanice, 40: 145-152.

Montesanto B, Ziller S, Coste M. 1999. Diatomées épilithiques et qualité biologique des ruisseaux du mont stratonikon, Chalkidiki (Grèce). Cryptogamie, Algo., 20(3): 235-251.
Morin S, Coste M. 2006. Atlas des Diatomées du Bassin Versant du Riou-Mort. Cemagref: Bordeaux; 109 p.

Nassali H, Ben bouih H, Srhiri A. 2002. Influence des Eaux sur la Dégradation de la Qualité des Eaux du lac Fouarat au Maroc. EPCOWN: Tunis; 3-4.

Pierre JF. 1994. Les Ried d'alsace: Essai d'une phytosociologie algale. Bulletin des Académie et Société Lorraines des Sciences, 33(3): 125-139.

Prygiel J, Carpentier P, Almeida S, Coste M, Druart J-C, Ector L, Guillard D, Honore MA, Iserentant R, Ledeganck P, LalanneCassou C, Lesniak C, Mercier I, Moncaut P, Nazart M, Nouchet N, Peres F, Peeters V, Rimet F, Rumeau A. 2002. Determination of the biological Diatom. Journal of Applied Phycology, 14: 27-39.

Rimet F, Matte JL, Mazuer P. 2006. Analyse de l'Application de Deux Indices Diatomées sur les Cours d'Eau Lorrains. I.B.D. et I.P.S.: Diren Lorraine; $21 \mathrm{p}$.

Rimet F, Heudre D, Matte JL, Mazuer P. 2006. Communauté de Diatomées des Bassins Moselle, Meuse et SarreCorrespondance avec les HydroEcorégions. Diren Lorraine; 51pp.

Rodier J. 1996. L'Analyse de l'Eau Naturelle, Eaux Résiduaires, Eau de Mer (8 ${ }^{\text {ème }}$ edn): Dunod, Paris: 1383 p.

Rumeau A, Coste M. 1988. Initiation à la systématique des Diatomées d'eau douce pour l'utilisation pratique d'un indice diatomique générique. Bulletin Fr. pêche et Piscic., 309: 69 p.

Stancheva R, Mancheva A, Ivanov P. 2007. Taxonomic composition of the epilithic diatom flora from rivers Vit and Osum, Bulgaria. Hytologia Balcanica, 13(1): 53-64.

Straub F, Pokorni B, Miserez J-J, Montandon P-E. 2003. Note algologique iii. Nuisances algales dans le jura suisse en 
2003. Bulletin de la Société Neuchâteloises des Sciences Naturelles, 127: 57-67.

Thi Thuy D, Coste M, Feurtet-Mazel A, Boudou A, Thi Phuong Quynh LE, Dinh Kim D. 2007. Les communautés de diatomées du périphiton: un outil d'évaluation de la qualité des eaux dans le fleuve rouge et les rivières de Nhué et de Tolich (Hanoi, Vietnam). Actes des JSIRAUF, Hanoi, 6-9 Novembre 2007.

Verrecchia E, Girard M, Gasse F, Demant A, Carbonel P, Bourcier M, Thi Maï B, Goiran J-P, Blanc P-F, Morhange C, Vecchi L. 2000. La mobilité des milieux littoraux de Cumes, Champs Phlégréens,
Campanie, Italie du Sud. Méditerrané, 94: 71-82.

Winter JG, Duthie HC. 2000. Epilithic diatoms as indicators of stream total $\mathrm{N}$ and total P concentration. Journal of the North American Benthological Society, 19: $32-49$.

Wunsam S, Cattaneo A, Bourassa N. 2002. Comparing diatom species, genera and size in biomonitoring: a case study from streams in the Laurentians (Quebec, Canada). Freshwater Biology, 47: 325340 . 